\title{
Epithelioma cuniculatum arising on a preexisting wart
}

\section{Mrinal Gupta}

\author{
Consultant Dermatologist, Treatwell Skin Centre, Jammu, India
}

Corresponding author: Dr. Mrinal Gupta, E-mail: drmrinalgupta@yahoo.com

\begin{abstract}
Epithelioma cuniculatum is a rare, well-differentiated variant of verrucous carcinoma, arising almost exclusively on foot, with a minimal incidence of metastasis. Clinically, it presents as a non-verrucous, slow growing, exophytic plaque or tumor of the plantar region with a penetration in the deep tissues. Histological examination shows a proliferation of well-differentiated keratinocytes. Conservative treatment and local excision have been utilized, but high recurrence rates have been observed. We report a case of epithelioma cuniculatum arising on the plantar aspect of foot in a 60-year old male, which arose at the site of a preexisting wart, which was managed by surgical excision.
\end{abstract}

Key words: Epithelioma cuniculatum; Squamous cell carcinoma; Verrucous carcinoma; Carcinoma; Human papilloma virus

\section{INTRODUCTION}

Epithelioma cuniculatum refers to verrucous carcinoma found almost exclusively in the foot. It is a rare, welldifferentiated variant of squamous cell carcinoma. Its clinical presentations are variable, but usually it presents as a slow growing, exophytic, low-grade and with minimal dysplasia. The tumor rarely metastasizes but it is capable of a slow and progressive invasion of the deeper tissues, i.e. subcutaneous fat and bone [1]. Most commonly, it is unilateral but bilateral cases have also been reported [2]. Herein, we report a case of epithelioma cuniculatum arising on the plantar aspect of foot in a 60-year old male, which arose at the site of a preexisting wart.

\section{CASE REPORT}

A 60-year old male presented to us with the chief complaints of a gradually progressive, large exophytic growth with seropurulent discharge on the plantar aspect of the right foot for the last five years. The patient gave a history of a plantar wart at the same site for the last eight years for which he had undergone radiofrequency ablation twice. Presently, the lesion was gradually increasing in size and was associated with pain and blood stained discharge for the last one year. There was no history of prolonged fever, loss of appetite, weight loss, chronic cough, family or personal history of tuberculosis. Cutaneous examination revealed a single verrucous lesion measuring $3 \times 3 \mathrm{~cm}$ on the plantar aspect of right foot associated with a blood stained discharge (Fig. 1). There was no associated lymphadenopathy and the systemic examination was also normal. Routine hematological, biochemical and serological investigations were within normal limits. $\mathrm{X}$-ray examination of the right foot revealed soft tissue swelling. Mantoux's test was negative and chest X-ray was also normal.

The differential diagnoses of tuberculosis verrucosa cutis, epithelioma cuniculatum and giant plantar wart were considered and a marginal incisonal biopsy was performed. Histopathology of the lesion revealed hyperkeratosis, papillomatosis, parakeratosis and elongated rete ridges with keratinocyte hyperplasia. There was formation of multiple large keratin-filled cysts and crypts with burrow like invaginations. The keratinocytes appeared well differentiated without any signs of atypia or loss of polarity. The histological features confirmed the diagnosis of epithelioma cuniculatum and the patient was advised a wide surgical 


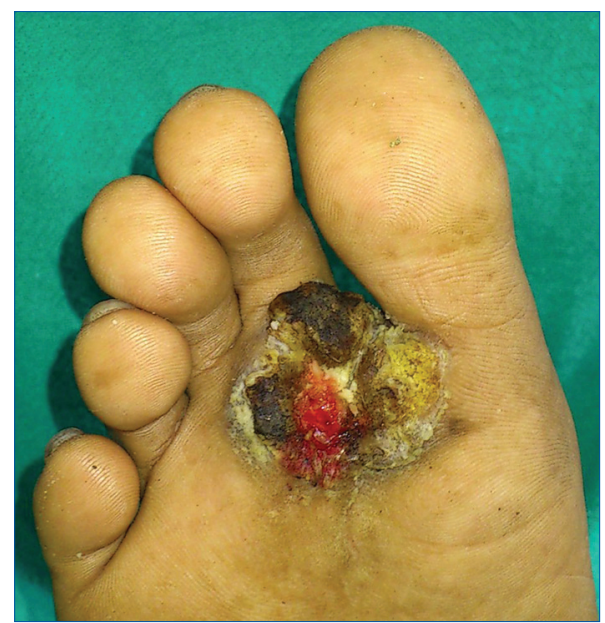

Figure 1: Large exophytic growth on the plantar aspect of the foot.

excision. Regular post-surgery follow up was done for six months and the patient remained well with no tumor recurrence.

\section{DISCUSSION}

Epithelioma cuniculatum, first described in 1954 by Professor Ian Aird, refers to verrucous carcinoma found almost exclusively in the foot. It is rare tumor with only about 100 cases reported so far. It occurs in older patients, usually during the $5^{\text {th }}$-6th decade, and with a higher prevalence in men as compared to women [2]. Etiolgically, chronic and repeated plantar trauma, slowly repairing bone fracture or osteomyelitis, local infiltration of corticosteroids, chronic decubitus ulcer and chronic inflammatory diseases have been hypothesized to play a role in its development $[2,4]$. Chronic infection by human papillomavirus 1-4, 6, 11 and 18 has also been hypothesized to play a role in pathogenesis due to their weak oncogenic potential.

The gross clinical appearance of epithelioma cuniculatum is characteristic and distinctive, presenting as a warty, keratotic tumor with crypts and sinuses that drain a malodorous exudate. The lesion is almost always single and bilateral lesions have been described only in a few patients. The anterior weight bearing area of the foot is more commonly involved than the heel or the arch [3]. The diagnosis is usually made at a later stage as the lesion is very often misdiagnosed at first as a wart or corn, which grows progressively despite topical treatments. The tumor rarely metastasizes but the delay in diagnosis allows the invasion to the underlying bones $[1,2,4]$.
Histopathology of the tumor is characterized by a well-differentiated squamous cell carcinoma with lowgrade cytological atypia and burrowing sinus tracts, often filled with keratinous debris, descending to the subcutaneous fat and sometimes infiltrating the bone [4].

Patients with verrucous carcinoma usually have a favorable prognosis with 5-year survival rates of $75 \%$. Wide local excision with at least a 5 -mm tumor-free margin remains the treatment of choice. Other modalities like electrodessication, cryotherapy, and laser ablation, are not advisable as they often result in tumor recurrence. Amputation is necessary when the tumor is too extensive or recurs after multiple attempts of local excision $[1,2]$.

In conclusion, epithelioma cuniculatum is a rare tumor with low intrinsic metastatic potential. However, it is often misdiagnosed which may lead to invasion of the underlying bone which requires amputation, thus leading to substantial morbidity. Therefore dermatologists should be aware of the condition and should have a better knowledge of this uncommon carcinoma.

\section{CONSENT}

The examination of the patient was conducted according to the Declaration of Helsinki principles.

\section{REFERENCES}

1. Ray R, Bhagat A, Vasudevan B, Sridhar J, Madan R, Ray M. A rare case of plantar epithelioma cuniculatum arising from a wart. Indian J Dermatol. 2015;60:485-7.

2. Arisi M, Zane C, Edu I, Battocchio S, Petrilli G, CalzavaraPinton PG. Carcinoma Cuniculatum of the Foot Invading the Bone Mimicking a Pseudo-Epitheliomatous Reaction to an Acute Osteomyelitis. Dermatol Ther (Heidelb). 2016;6:95-9.

3. Miller SB, Brandes BA, Mahmarian RR, Durham JR. Verrucous carcinoma of the foot: a review and report of two cases. J Foot Ankle Surg. 2001;40:225-31.

4. Schell BJ, Rosen T, Rady P, Arany I, Tschen JA, Mack MF, et al. Verrucous carcinoma of the foot associated with human papillomavirus type 16. J Am Acad Dermatol. 2001;45:49-55.

Copyright by Mrinal Gupta. This is an open-access article distributed under the terms of the Creative Commons Attribution License, which permits unrestricted use, distribution, and reproduction in any medium, provided the original author and source are credited.

Source of Support: Nil, Conflict of Interest: None declared. 